\title{
PROGRESSION OF DIABETIC RETINOPATHY AND RUBEOTIC GLAUCOMA FOLLOWING CATARACT SURGERY
}

\author{
S. A. SADIQ ${ }^{1}$, A. CHATTERJEE ${ }^{2}$ and S. A. VERNON ${ }^{1}$ \\ Nottingham and Manchester
}

\begin{abstract}
SUMMARY
There has been recent interest in the progression of diabetic retinopathy following extracapsular cataract extraction (ECCE) especially with vitreous loss. It is well known that diabetic retinopathy progresses after intracapsular cataract extraction (ICCE) but was thought to be less common after ECCE. We present 7 patients with symmetrical non-proliferative diabetic retinopathy who underwent ECCE with intraocular lens (IOL) implantation. These patients ranged in age from 56 to 69 years; 2 were insulin-dependent diabetics (IDDM) and 5 non-IDDMs. Rubeosis iridis developed quickly between post-operative outpatient visits despite good diabetic control and a static retinal picture in the fellow eye. Visual loss following the onset of rubeosis was severe, with 3 patients needing cyclocryotherapy and eventually having no perception of light. The rapid onset of rubeosis between post-operative outpatient visits leads us to suggest much shorter periods between reviews than is current practice and the consideration of routine panretinal photocoagulation in the immediate post-operative period in diabetics with worsening retinopathy after ECCE and IOL. Possible causes of the increase in neovascularisation and rubeosis are discussed. The most important message highlighted by these case histories is that the surgery and follow-up of diabetic patients undergoing surgery should be undertaken by an ophthalmologist with an interest in diabetes. Where there is no recognised diabetic retinal specialist in a unit, then early referral to such an ophthalmologist is recommended when complications arise.
\end{abstract}

There have been reports of complications of cataract surgery in diabetics, and of progression of diabetic

From: ${ }^{1}$ Queen's Medical Centre, Nottingham; ${ }^{2}$ Royal Eye Hospital, Manchester, UK.

Correspondence to: Mr S. A. Sadiq, Ophthalmology Department, Queens Medical Centre, Nottingham NG7 2UH, UK. retinopathy following cataract surgery over a prolonged time period compared with the fellow eye which initially had a similar level of retinopathy. ${ }^{1,2}$ Recently two cases have been reported of rapid progression of retinopathy in the immediate post-operative period with rubeosis developing within a few weeks of surgery in the operated eye only. ${ }^{3}$

We present 7 cases of diabetics who progressed to aggressive neovascularisation or rubeosis leading to rubeotic glaucoma and severe visual loss within a few weeks of surgery. These patients were managed by general ophthalmologists in centres where there were no consultants with an interest in diabetes (except one), and nor were these patients referred to a recognised diabetic specialist when complications arose.

\section{CASE REPORTS}

Case 1

A 65-year-old West Indian man with a 26 year history of non-insulin-dependent diabetes mellitus (non-IDDM) presented in August 1989 with a right visual acuity (VA) of $6 / 36$ and a left VA of $6 / 60$. He was found to have bilateral posterior subcapsular and cortical cataracts. Fundal examination revealed exudates at the right macula, and two incomplete circinate rings around the left macula. There were no new vessels (NV) in either eye. Focal laser was not performed for the left circinate rings as it was felt that the fundal view was inadequate.

He underwent an uncomplicated left extracapsular cataract extraction (ECCE) with intraocular lens implantation (IOL) in November 1989. Four months post-operatively his VA was $6 / 60$ in the right eye and $6 / 18$ in the left with new spectacles. The intraocular pressure (IOP) was $20 \mathrm{mmHg}$ in each eye. Fundal 
examination revealed right-sided maculopathy and a left incomplete circinate ring inferior to the macula. At this stage, the consultant felt that the retinopathy did not merit laser treatment, but the patient was placed on the waiting list for right ECCE.

In May 1990 the patient presented with a 1 month history of the left eye being increasingly painful and red. The left VA had reduced to counting fingers $(\mathrm{CF})$, and was very injected with rubeosis iridis and an IOP of $44 \mathrm{mmHg}$. Fundal examination showed the left venous system not to be engorged, and there were no NVs. Gonioscopy showed early NVs in the drainage angle. He underwent an urgent left panretinal photocoagulation (PRP) with only 677 burns due to a poor view. A month later his left VA was no perception of light (NPL) with frank rubeosis iridis and corneal oedema, with an IOP of $70 \mathrm{mmHg}$ despite treatment with timolol, betamethasone and atropine drops, and acetazolamide systemically. He was in pain and underwent left inferior half cyclocryotherapy, later followed by superior half cyclocryotherapy. After this his left eye became painfree with a VA of NPL and an IOP around 40 $\mathrm{mmHg}$.

During this period the right VA had worsened to $\mathrm{CF}$ due to increasing cataract. Right fundus again revealed a maculopathy, but there were no retinal or disc NVs. Understandably, he was not keen to proceed with right ECCE.

\section{Case 2}

A 69-year-old man with non-IDDM presented in August 1987 with a right VA of 6/60 and left VA of $6 / 18$. Fundus examination revealed bilateral early diabetic maculopathy. He underwent argon laser photocoagulation to a left circinate ring in November 1987 but the left VA decreased to CF due to increasing maculopathy by February 1988. A year later the right VA had also reduced to CF due to a combination of posterior subcapsular cataract and maculopathy. In June 1990 he underwent an uncomplicated right ECCE with IOL. Post-operatively the VA improved only to $6 / 60$ in the right eye. The IOPs were $10 \mathrm{mmHg}$ in the right eye and 14 $\mathrm{mmHg}$ in the left. Fundal examination revealed the bilateral maculopathy, but with no NVs. A fundal fluorescein angiogram (FFA) was not obtained. In September 1990 he returned to the casualty department with a right VA of CF and an injected eye. The right cornea was oedematous with an inflamed anterior chamber and an IOP of $38 \mathrm{mmHg}$ in the right eye and $14 \mathrm{mmHg}$ in the left. Right iris rubeosis was seen but there were no retinal NVs.

The IOP responded partially to medical treatment as an inpatient, but 2 weeks after admission the patient became unwell and was referred to the diabetic ward where he later died.
Case 3

A 56-year-old woman with non-IDDM was first seen in 1981 with a VA of $6 / 9$ and IOPs of $16 \mathrm{mmHg}$ in both eyes; both fundi showed background retinopathy only. The situation was stable until 1986 when the VAs had reduced to $6 / 36$ in the right eye and $6 / 12$ in the left due to increasing cataract. In October 1986 she underwent an uncomplicated right ECCE with IOL. One month post-operatively the right VA had improved only to $6 / 36$ due to a thickened posterior capsule and early maculopathy, although a FFA was not requested. Two months postoperatively the right IOP was found to be elevated at $30 \mathrm{mmHg}$ (left IOP was $18 \mathrm{mmHg}$ ) and blood vessels were noted on the thickened posterior capsule. Both fundi showed macular exudates. Treatment was started with timolol drops $0.5 \%$ b.d. which reduced the right IOP to under $21 \mathrm{mmHg}$.

In February 1987 a right macular laser photocoagulation was performed for the maculopathy, whilst pilocarpine drops and acetazolamide tablets were added to try to control the IOP. By March 1987 the right VA was CF and rubeosis of the right iris was noted, whilst the left VA was 6/24. Over the next year the right IOP varied between $20 \mathrm{mmHg}$ and 22 $\mathrm{mmHg}$ whilst the left IOP varied between $14 \mathrm{mmHg}$ and $16 \mathrm{mmHg}$. Fundal examination revealed bilateral maculopathy, worse in the right eye.

In March 1988 the right VA decreased to hand movements (HM) due to vitreous haemorrhage. The right IOP was $30 \mathrm{mmHg}$ with a rubeotic iris. The left VA was $6 / 36$, with an IOP of $14 \mathrm{mmHg}$ and unchanged maculopathy. The right eye underwent three episodes of laser PRP (1043 burns) in April and May 1988. The right IOP remained elevated at $30 \mathrm{mmHg}$ despite medical treatment and so a right cyclocryotherapy was performed in June 1988, which resulted in a post-operative right IOP below 20 $\mathrm{mmHg}$.

Over the next 2 years the right eye had a further four episodes of vitreous haemorrhage due to disc $\mathrm{NVs}$, leaving a residual vision of $\mathrm{CF}$.

\section{Case 4}

A 57-year-old man with a 25 year history of IDDM presented in June 1990 with a gradual reduction of VA in both eyes over the previous 2 years. He was hypertensive and his regular medication consisted of atenolol, cyclopenthiazide and insulin. His VA was $\mathrm{CF}$ in both eyes and IOPs were $18 \mathrm{mmHg}$ in the right eye and $15 \mathrm{mmHg}$ in the left. He had bilateral posterior subcapsular cataract, but there was no diabetic retinopathy in either eye.

He underwent right ECCE under local anaesthesia in August 1990, which was complicated by posterior capsule rupture requiring anterior vitrectomy and 
insertion of an anterior chamber IOL. Post-operatively the right VA improved to 6/12 after refraction. No retinopathy was noted. A year later he underwent uncomplicated left ECCE with posterior chamber IOL. At this stage, his right VA was $6 / 24$ and left VA was 6/12, and IOPs were $9 \mathrm{mmHg}$ in the right eye and $11 \mathrm{mmHg}$ in the left eye.

In October 1991 he presented with a 3 month history of reduction in the right VA to CF. The left VA was 6/18. The right eye showed rubeosis of the iris. IOPs were $8 \mathrm{mmHg}$ in the right eye and 16 $\mathrm{mmHg}$ in the left. No diabetic retinopathy was seen in either eye, although the right fundal view was hazy. He underwent two sessions of right PRP (1965 burns) in the following 6 months and this resulted in a reduction in the iris rubeosis. In June 1992 his VA was $6 / 18$ in the right eye and $6 / 12$ in the left. IOPs were $25 \mathrm{mmHg}$ in the right eye and $14 \mathrm{mmHg}$ in the left. No diabetic retinopathy was seen in either eye and he was started on timolol drops $0.5 \%$ b.d. to the right eye with good effect.

\section{Case 5}

A 64-year-old man with a 15 year history of nonIDDM treated with metformin was referred for diabetic retinopathy screening. His VA was $6 / 18$ in the right eye and 6/9 in the left eye, and IOPs were 21 $\mathrm{mmHg}$ in both eyes. He had right-sided posterior subcapsular cataract. Fundal examination revealed bilateral background diabetic retinopathy. In the following 2 years he required laser treatment for circinate rings: on one occasion to the right eye and on three occasions to the left eye.

Four years after presentation, the right VA was further reduced to $\mathrm{CF}$ due to increasing cataract. The left VA was $6 / 12$ and the IOPs were $19 \mathrm{mmHg}$ in both eyes. Fundal examination revealed bilateral background retinopathy with regression of circinate rings. He underwent planned right ICCE and anterior chamber IOL in October 1988. Although there was an IOP spike on the first post-operative day, this settled with conservative treatment. Three weeks later, the right VA had improved to 6/36, whilst the left VA was $6 / 18$. IOPs were $34 \mathrm{mmHg}$ in the right eye and $20 \mathrm{mmHg}$ in the left eye, and the patient was commenced on timolol drops to the right eye. Fundal examination revealed some macular exudates in the right eye, but there was no evidence of neovascularisation. The left eye showed background diabetic retinopathy at that time. Over the next 3 months the right IOP continued to be raised, and so systemic acetazolamide was added; this reduced the right IOP to under $20 \mathrm{mmHg}$ until November 1989, when he presented with right-sided floaters due to a vitreous haemorrhage. His IOPs were $40 \mathrm{mmHg}$ in the right eye and $20 \mathrm{mmHg}$ in the left eye. In March 1990 the right visual acuity was
$\mathrm{CF}$, the right iris was rubeotic and the cornea was oedematous. IOPs at this stage were $55 \mathrm{mHg}$ in the right eye and $12 \mathrm{mmHg}$ in the left eye. He underwent right cyclocryotherapy which made the right eye comfortable. By June 1991 the right VA was NPL.

In October 1992 the left vision had reduced to $6 / 60$ due to increasing cataract, and the patient underwent uncomplicated left ECCE with posterior chamber IOL which improved the left vision to 6/24. Postoperatively, the left IOP was $18 \mathrm{mmHg}$, and fundal examination revealed background diabetic retinopathy without any NVs. However, by December 1992 the left IOP had risen to $25 \mathrm{mmHg}$, and although no iris vessels were seen, NVs were found in the drainage angle and on the left optic disc. He underwent a left PRP (858 burns) which resulted in regression of the disc NVs, leaving a VA of 6/18 in his left and only eye. In January 1993 the left IOP was $25 \mathrm{mmHg}$ and early disc cupping was noted. $\mathrm{He}$ underwent a left trabeculectomy which unfortunately failed, and medical treatment was reinstituted. He underwent two further episodes of left PRP for mild recurrence of the disc NVs with a good result. At his latest clinic visit the right eye was comfortable, and his VA was NPL in the right eye and 6/18 in the left eye. IOPs were $40 \mathrm{mmHg}$ in the right eye and 18 $\mathrm{mmHg}$ in the left eye. There were no NVs in the left fundus.

\section{Case 6}

A 66-year-old man with a 1 year history of nonIDDM and hypertension treated with gliclazide, metformin and nifedipine retard, presented in September 1993 with a reduction in left vision. His VA was 6/12 in the right eye and CF in the left eye, and his IOPs were $21 \mathrm{mmHg}$ in each eye. He had a moderate left cataract and there was no evidence of diabetic retinopathy in either eye. In January 1994 he underwent an uncomplicated left ECCE and IOL. One month post-operatively the left vision was $6 / 60$ unaided and the left eye was quiet, with an IOP of 15 $\mathrm{mmHg}$. The left fundus revealed some cystoid macular oedema but no retinopathy, although a FFA was not performed. When seen in April 1994, the VA was $6 / 9$ in the right eye and CF in the left eye. The left IOP had risen to $40 \mathrm{mmHg}$ and the left anterior chamber was quiet. The patient was found to have early rubeosis of the left iris. Gonioscopy revealed NVs in the drainage angle. The left optic disc showed early thinning of the inferior rim, but there were no signs of neovascularisation. There was persistent cystoid macular oedema. He was started on carteolol drops and underwent a left PRP (760 burns), following which the left IOP was initially under $20 \mathrm{mmHg}$ without any topical therapy. Two months later it had again risen to $40 \mathrm{mmHg}$, and so carteolol 1\% b.d. was restarted. At his last clinic visit, 
his VA was $6 / 12$ in the right eye and $6 / 60$ in the left eye. IOPs were $18 \mathrm{mmHg}$ in the right eye and 23 $\mathrm{mmHg}$ in the left eye. There was no diabetic retinopathy in either eye.

\section{Case 7}

A 57-year-old man presented in March 1991 with an 8 year history of non-IDDM controlled with gliclazide. His VA was $6 / 6$ in both eyes, IOPs were $18 \mathrm{mmHg}$ in each eye, and there were early bilateral cortical cataracts. Both fundi showed mild background diabetic retinopathy in the form of microaneurysms and dot haemorrhages. There were no exudates, cotton wool spots or NVs. In July 1993 he underwent a right macular grid photocoagulation for oedema. By June 1994 his VA had deteriorated to $6 / 60$ in the right eye and 6/18 in the left eye, and he underwent an uncomplicated right ECCE with IOL under local anaesthesia. However, at 3 weeks postoperatively he was thought to be developing early rubeosis although the IOP remained normal at 20 $\mathrm{mmHg}$. The right posterior pole showed some macular oedema, but there were no signs of NVs. He was booked for PRP but failed to attend for this. When seen at 11 weeks he had developed frank rubeosis with an IOP of $40 \mathrm{mmHg}$ in the right eye. The left IOP was $18 \mathrm{mmHg}$. His VA at this stage was $\mathrm{CF}$ in the right eye and 6/24 in the left eye. The right macula revealed some oedema and the disc was pale, but there were no discs NVs.

\section{DISCUSSION}

There have been previous reports of increases in diabetic retinopathy following cataract surgery. ${ }^{4,5}$ However, reports of rubeotic glaucoma following cataract surgery in diabetics are few and involve small numbers. ${ }^{3,4}$ Jaffe et al..$^{5}$ showed that cataract extraction was highly associated with asymmetrical progression of non-proliferative retinopathy, with progression in $74 \%$ of operated eyes. No patient had progression in the unoperated eye alone.

Our cases repeat this finding, all showing progression of retinopathy in the operated eye only and rapid progression of the proliferative changes leading to rubeosis iridis and neovascular glaucoma within a few months of surgery (range 1-18 months).

Possible mechanisms of progression of diabetic retinopathy and rubeosis remain to be determined. Surgical trauma may contribute to the breakdown of the blood-retina barrier leading to increases in macular oedema, haemorrhages and exudates. ${ }^{5}$ Successful phacoemulsification causes less surgical trauma and less post-operative inflammation. It would seem the best method of cataract surgery in diabetic patients, although as yet no controlled study has investigated this.

An intact posterior capsule may protect against forward migration of any retinal angiogenic factor and also reduce the backward flow of an agent postulated to cause post-operative cystoid macular oedema. This would explain the increased complications following intracapsular surgery or in cases where posterior capsule rupture occurs.

We recommend that, where dense cataract precludes visualisation of the fundus, cataract surgery should be undertaken although with a guarded prognosis, as assessment and treatment of any retinopathy may otherwise be impossible.

Since surgery may lead to aggravation of diabetic retinopathy it is preferable to delay surgery in a patient with background diabetic retinopathy for as long as he or she is able to cope with their daily activities, although IDDM patients may require earlier surgery if the cataract is interfering with accurate administration of their medication. This has become less of a problem since the introduction of insulin pens where insulin does not have to be drawn up by the patient.

Patients with pre-operative diabetic retinopathy should be advised that the visual prognosis for cataract extraction is worse than it would be if no retinopathy were present. Those with early or moderate cataract and diabetic retinopathy, particularly proliferative, should be treated pre-operatively, with cataract extraction being performed when neovascularisation has receded. If cataract precludes adequate laser treatment, then this can be performed immediately after cataract extraction with an indirect laser system while the patient is still on the operating table or early on in the post-operative period.

Particular care should be taken to maintain an intact posterior capsule during surgery to retain its barrier effect against forward diffusion of any angiogenic factor, or backward movement of a postulated agent causing cystoid macular oedema.

The key message we would like to highlight from these case reports is that the surgery and follow-up of these patients should be undertaken by an ophthalmologist with an interest in diabetes. In such a setting, the optimum use can also be made of fluorescein angiography for those patients who seem to be running into problems, so that significant changes are identified at an early stage and appropriate treatment given. Where there is no recognised diabetic retinal specialist in a unit, then early referral to such a specialist is recommended before complications progress.

Key words: Cataract surgery, Diabetes, Glaucoma, Retinopathy, Rubeosis.

\section{REFERENCES}

1. Jaffe GJ, Burton TC. Progression of nonproliferative diabetic retinopathy following cataract extraction. Arch Ophthalmol 1988;106:745.

2. Pollack A, Dotan S, Oliver M. Progression of diabetic 
retinopathy after cataract extraction. $\mathrm{Br} \mathrm{J}$ Ophthalmol 1991;75:547.

3. Prasad P, Setna PH, Dunne JA. Accelerated ocular neovascularisation in diabetics following posterior chamber lens implantation. $\mathrm{Br} \mathrm{J}$ Ophthalmol 1990;74:313-4.

4. Benson WE, Brown GC, Tasman W, McNamara JA, Vander JF. Extracapsular cataract extraction and placement of a posterior chamber lens in patients with diabetic retinopathy. Ophthalmology 1993;100:730-8.

5. Jaffe GJ, Burton TC, Kuhn E, Prestcott A, Hartz A. Progression of non-proliferative retinopathy and visual outcome after extracapsular cataract extraction and intraocular lens implantation. Am J Ophthalmol 1992;114:448-56.

6. Alphar JJ. Cataract extraction and diabetic retinopathy. Am Intraocular Implant Soc 1984;10:433. 